Research Paper

\title{
The Efficacy of Upfront Intracranial Radiation with TKI Compared to TKI Alone in the NSCLC Patients Harboring EGFR Mutation and Brain Metastases
}

Chunyu Wang1, Xiaotong Lu1, Zongmei Zhou'1, Jingbo Wang1, Zhouguang Hui1 2, Jun Liang1, QinFu Feng1, Dongfu Chen ${ }^{1}$, Zefen Xiao ${ }^{1}$, Jima Lv¹, Xiaozhen Wang1, Xin Wang1, Tao Zhang1, Lei Deng1, Wenqing Wang ${ }^{1}$, Jianping $\mathrm{Xiao}^{1}$, Junling $\mathrm{Li}^{3}$, Nan $\mathrm{Bi}^{1}{ }^{\circledR}$, Luhua Wang ${ }^{\circledR}$

1. Department of Radiation Oncology, National Cancer Center/Cancer Hospital, Chinese Academy of Medical Sciences and Peking Union Medical College, Beijing, People's Republic of China, 100021

2. Department of VIP Medical Services, National Cancer Center/ Cancer Hospital, Chinese Academy of Medical Sciences and Peking Union Medical College, Beijing, China, 100021

3. Department of Medicine Oncology, National Cancer Center/Cancer Hospital, Chinese Academy of Medical Sciences and Peking Union Medical College, Beijing, People's Republic of China, 100021

$\square$ Corresponding authors: Luhua Wang, No.17 Panjiayuannanli, Chaoyang District, Beijing, People's Republic of China, 100021. E-mail: wlhwq@yahoo.com, Tel: +8610 87788799. Nan Bi, No.17 Panjiayuannanli, Chaoyang District, Beijing, People's Republic of China, 100021. E-mail: binan_email@163.com, Tel: +8610 87788995

(c) Ivyspring International Publisher. This is an open access article distributed under the terms of the Creative Commons Attribution (CC BY-NC) license (https://creativecommons.org/licenses/by-nc/4.0/). See http://ivyspring.com/terms for full terms and conditions.

Received: 2018.09.22; Accepted: 2019.04.14; Published: 2019.05.12

\begin{abstract}
Introduction: The high intracranial efficacy of EGFR-TKI challenges the role of upfront intracranial radiation therapy (RT) in non-small cell lung cancer (NSCLC) patients with EGFR mutation and brain metastases (BM). Therefore, we conducted a retrospective analysis to demonstrate the role of upfront RT in these patients.

Materials and Methods: Patients that had histologically confirmed NSCLC with EGFR mutation, brain metastases, and received TKI or upfront RT with TKI were included in this study. Intracranial progression was estimated using the Fine-Gray competing risks model. Kaplan-Meier analysis and Log-rank test were used to evaluate and compare intracranial progression-free survival (iPFS), systemic PFS (sPFS), time to second-line systematic therapy (SST) and overall survival (OS).

Results: Among the 93 patients included, 53 patients received upfront RT and TKI, and 40 patients received TKI only. Upfront RT group showed lower intracranial progression risk with adjusted SHR $0.38(95 \% \mathrm{Cl}, 0.19$ to $0.75, P=0.006)$ and longer median time to sPFS (15.6 vs 8.9 months, $P=0.009)$. There were 9 out of $36(25 \%)$ and 16 out of 34 (47.1\%) patients who had oligo-progression received salvage RT in the RT group and TKI group, respectively. After the salvage RT, upfront RT did not prolong the median time to SST ( 23.6 vs 18.9 months, $P=0.862$ ) and OS (median time, 35.4 vs 35.8 months, $P=0.695$ ) compared to TKI alone.

Conclusion: Compared to upfront intracranial RT, the salvage RT to oligo-progressive disease allowed patients getting TKI to have similar time on initial TKI and OS despite worse iPFS. The best timing of intracranial RT remains to be further verified.
\end{abstract}

Key words: non-small cell lung cancer, EGFR mutation, brain metastases, radiation, tyrosine kinase inhibitors

\section{Introduction}

Lung cancer has the highest mortality among all cancers around the world[1, 2]. The non-small cell lung cancer (NSCLC) comprises more than $80 \%$ of lung cancers, and almost $50 \%$ of NSCLC are lung adenocarcinoma[3]. About $30 \%$ of stage IV NSCLC patients present with brain metastases $(\mathrm{BM})$ at the 
time of diagnosis[4]. The median overall survival (OS) varies from 3.0-14.8 months according to their graded prognostic assessment (GPA) scores[5]. Whole-brain radiotherapy (WBRT), stereotactic radiosurgery (SRS), or surgical resection, used alone or in combination, is the first line treatment for BM. While therapy to the brain relieves the intracranial symptom and improves the intracranial local control, it may cause neurocognitive toxicity that presents later in the course[6, 7].

The discovery of EGFR mutation introduced new treatment hope for NSCLC. The invention of EGFR-tyrosine kinase inhibitors (TKI) drastically altered the clinical evaluation and treatment options for NSCLC patients. Patients with EGFR mutation have a $50-70 \%$ risk for developing BM[8]. A few phase II clinical studies reported that single-agent EGFR-TKI showed promising results in TKI treated-naive patients. The intracranial response rate (iRR) was $75 \%-88 \%$; median intracranial progression-free survival (iPFS) was 6.6-14.5 months, and median OS was 15.9-21.8 months [9-11]. Subsequently, the question is whether there is enough evidence to defer radiation therapy (RT) until the intracranial progression on TKI. Regrettably, no published phase III clinical trial answered this question. Therefore, we conducted a retrospective analysis to compare the upfront RT role in the treatment of NSCLC harboring EGFR mutation and BM.

\section{Materials and Methods}

\section{Patients}

The institutional review board at our institution approved the present study. We retrospectively reviewed NSCLC patients treated at our institution from January of 2010 to December of 2016. All patients included in this study met the following criteria: 1) histologically confirmed NSCLC; 2) EGFR-TKI sensitive mutation confirmed by polymerase chain reaction amplification (PCR); 3) MRI confirmed brain metastases; 4) treated with TKI or upfront RT concurrently with/followed by TKI; 5) complete pretreatment baseline data. The exclusion criteria included: 1) prior EGFR-TKI treatment before brain metastases; 2) no follow-up data achievable; 3) Synchronous or metachronous malignancies (except for cutaneous (non-melanomas) carcinoma, thyroid papillary carcinoma, phase I seminoma or cervical carcinoma in situ that were curatively treated).

The following variables were reviewed for analyses: date of birth, gender, smoking history, the Karnofsky Performance Score (KPS) when BM was diagnosed, histology of NSCLC, EGFR mutation type, stage, $\mathrm{BM}$ diagnose date, number of brain metastases, maximum size of $\mathrm{BM}$, presence of $\mathrm{BM}$ symptom, extracranial metastases status, and the name of EGFR-TKI. The disease-specific Graded Prognostic Assessment (ds-GPA) was calculated according to the published study[5]. The intracranial lesions were evaluated every three months by magnetic resonance imaging (MRI), and the primary lesion and other metastasis sites were monitored by computed tomography (CT) or positron emission tomography-CT if needed every three months. The intracranial objective response rate (iORR) was evaluated using The Response Evaluation Criteria in Solid Tumors (RECIST, version 1.1) [12]. The first progression site and date were recorded with intracranial (primary BM, new lesion or both), extracranial or concurrent failure. The treatment regimens after any progression were documented. Of note, the dates patients started to receive a second-line systematic therapy (SST) were recorded. The most recent follow-up time was recorded.

\section{Statistical Analysis}

The patients' characteristics and iORR in the upfront RT group and TKI alone group were compared with the $\chi^{2}$ test or Fisher's exact for categorical variables, and one-way analysis of variance for continuous data. The iPFS was calculated from the date of $\mathrm{BM}$ diagnoses to the progression of the primary metastases sites or a new intracranial lesion or both. The sPFS was derived from the date of $\mathrm{BM}$ diagnoses to the progress of any site, whether intracranial or extracranial. The time to SST was calculated from the date of $\mathrm{BM}$ diagnoses to the initiation of a second-line systematic therapy to the whole body. The OS was derived from the date of brain metastases until the date of death or censored on the last follow up. Kaplan-Meier analysis was used to estimate iPFS, systemic PFS (sPFS), time to SST and OS. The log-rank test was used to compare the data. Considering the competing risk of death to iPFS, we used univariable and multivariable Fine-Grey competing risk regression to compare the cumulative incidence rate of intracranial recurrence rate[13]. Univariable and multivariable Cox proportional hazards analysis examined factors associated with increased risk of death. Significance for inclusion in the multivariate model was set at $p<0.10$ and $p<0.05$ as a significant predictor of outcomes. Statistical analyses were performed using STATA 14 (Stata, College Station, TX).

\section{Results}

\section{Patients' selection and characteristics}

Between January 1st, 2010 and December 31 2016, 1499 NSCLC patients developed BM and 806 of 
them underwent an EGFR mutation test, among which 306 patients were identified with EGFR-mutated NSCLC with BM. Forty patients were excluded because they did not receive TKI therapy, and 57 patients were excluded due to development of BM after TKI treatment. Seventy-two patients were excluded because TKI was not the first line of treatment after the diagnosis of BM. Nine patients were excluded with a non-sensitizing EGFR mutation; 11 patients were excluded due to the concurrent metastases with leptomeningeal metastases; five patients were excluded due to simultaneous diagnosis with other cancers; 19 patients failed to follow-up in our institution. The remaining 93 patients were treated with RT and TKI (RT group, $\mathrm{n}=53$ ) or TKI alone (TKI group, $\mathrm{n}=40$ ). In the RT group, there are 30 , 14 and 9 patients received WBRT, SRS, and WBRT with simultaneously integrated boost treatment, respectively. Table 1 shows the baseline characteristics of the patients according to treatment groups. Most patients were never-smokers (63\%). Patients who received RT were more likely to have $\mathrm{BM}>10 \mathrm{~mm}(75 \%$ vs $57 \%, P=0.066)$ and had more symptomatic brain metastases ( $38 \%$ vs $20 \%, P=0.065)$. In the RT group, $32 \%$ of patients were stage I-III at diagnoses, with a lower percentage of patients in the TKI group (18\%). There was no difference between the two groups concerning age, gender, KPS, smoking history, number of BM, extracranial metastases, ds-GPA, EGFR mutation and type of TKI.

\section{Treatment outcome}

The intracranial lesion response rates were evaluated every three months. The results showed that $3(5.7 \%)$ patients had a complete response (CR), $47(88.7 \%)$ patients showed a partial response (PR), and $3(5.7 \%)$ patients had stable disease (SD) in upfront RT group. The corresponding numbers in the TKI alone group were 5 (12.5\%), $28(70 \%)$ and $6(15 \%)$, respectively. One $(2.5 \%)$ patient experienced disease progression in the TKI group. The upfront RT group showed a trend to have higher iORR compared to TKI alone $(94.3 \%$ vs $82.5 \%, P=0.093)$.

The median follow-up time for all patients was 37.7 months (range, 3.4-63.2). 23 (43.4\%) patients in the RT group and $25(62.5 \%)$ patients in the TKI group developed intracranial failure. The median iPFS for the entire cohort was 22.6 months (95\% confidence interval [CI], 13.6 - 31.6 months). The median iPFS for the RT group and the TKI group were 27.6 months (95\% CI 20.2 - 35.0 months) and 16.1 months (95\% CI 14.6 -17.6 months), respectively (log-rank $P=0.053$ ). After controlling for stage and number of metastases with the Fine-Gray competing risks regression model, upfront RT group showed a significantly lower probability of intracranial progression, with adjusted subdistribution hazard ratios (SHR) of 0.38 (95\% CI, 0.19 to $0.75 ; P=0.006$, Fig 1 , Table S1).

Table 1. Patient Characteristics

\begin{tabular}{|c|c|c|c|}
\hline & $\mathrm{RT}(\mathrm{n}=53, \%)$ & $\operatorname{TKI}(\mathrm{n}=40, \%)$ & $P$ \\
\hline \multicolumn{4}{|l|}{ Age at BM (years) } \\
\hline$>60$ & $20(38)$ & $18(45)$ & \multirow[t]{3}{*}{0.331} \\
\hline $50-60$ & $15(28)$ & $14(35)$ & \\
\hline$<50$ & $18(34)$ & $8(20)$ & \\
\hline \multicolumn{4}{|l|}{ Gender } \\
\hline Male & $25(47)$ & $19(48)$ & \multirow[t]{2}{*}{0.975} \\
\hline Female & $28(53)$ & $21(52)$ & \\
\hline \multicolumn{4}{|l|}{ KPS } \\
\hline $70-80$ & $43(81)$ & $28(70)$ & \multirow[t]{2}{*}{0.211} \\
\hline $90-100$ & $10(19)$ & $12(30)$ & \\
\hline \multicolumn{4}{|l|}{ Smoking history } \\
\hline No & $35(66)$ & $24(60)$ & \multirow[t]{2}{*}{$0.54 \mathrm{C}$} \\
\hline Yes & $18(34)$ & $16(40)$ & \\
\hline \multicolumn{4}{|l|}{ Number of BMs } \\
\hline $1-3$ & $24(45)$ & $22(55)$ & \multirow[t]{3}{*}{0.645} \\
\hline $4-10$ & $23(43)$ & $14(35)$ & \\
\hline$>10$ & $6(12)$ & $4(10)$ & \\
\hline \multicolumn{4}{|c|}{$\begin{array}{l}\text { Extracranial metastases at the time of } \\
\text { BM }\end{array}$} \\
\hline Yes & $30(55)$ & $28(70)$ & \multirow[t]{2}{*}{0.187} \\
\hline No & $23(45)$ & $12(30)$ & \\
\hline \multicolumn{4}{|l|}{ Stage at diagnose } \\
\hline I-III & $17(32)$ & $7(18)$ & \multirow[t]{2}{*}{0.112} \\
\hline IV & $36(68)$ & $33(82)$ & \\
\hline \multicolumn{4}{|l|}{ Largest size of BM } \\
\hline$\leq 10 \mathrm{~mm}$ & $13(25)$ & $17(43)$ & \multirow[t]{2}{*}{0.066} \\
\hline$>10 \mathrm{~mm}$ & $40(75)$ & $23(57)$ & \\
\hline \multicolumn{4}{|l|}{ Disease-specific GPA } \\
\hline $0-1.5$ & $24(45)$ & $21(53)$ & \multirow[t]{2}{*}{0.491} \\
\hline $2.0-4.0$ & $29(55)$ & $19(47)$ & \\
\hline \multicolumn{4}{|l|}{ Symptom from BM } \\
\hline No & $33(62)$ & $32(80)$ & \multirow[t]{2}{*}{0.065} \\
\hline Yes & $20(38)$ & $8(20)$ & \\
\hline \multicolumn{4}{|l|}{ EGFR mutation } \\
\hline Exon 19 deletion & $28(53)$ & $21(53)$ & \multirow[t]{2}{*}{0.975} \\
\hline Exon 21 L858R and others & $25(47)$ & $19(47)$ & \\
\hline \multicolumn{4}{|l|}{ EGFR-TKI } \\
\hline Gefitinib & $34(64)$ & $21(53)$ & \multirow[t]{4}{*}{0.288} \\
\hline Erlotinib & $12(23)$ & $8(20)$ & \\
\hline Afatinib & $0(0)$ & $1(2)$ & \\
\hline Icotinib & $7(13)$ & $10(25)$ & \\
\hline \multicolumn{4}{|c|}{ Use Osimertinib as salvage treatment } \\
\hline No & $44(83)$ & $32(80)$ & 0.709 \\
\hline Yes & $9(17)$ & $8(20)$ & \\
\hline
\end{tabular}

Until the last follow up, 70 out of 93 (75.3\%) patients experienced systematic progression. The RT group had longer sPFS (median time, 15.6 months, 95\% CI 4.9-26.4 months) than did the TKI group (median time, 8.9 months, 95\% CI 7.2-10.6 months) $(P=0.009$, Fig 2). As shown in Table 2, 36 and 34 patients developed failure in the RT and the TKI group, respectively. The numbers of the first site for intracranial, extracranial and simultaneous failure in RT group are $10(18 \%), 22(42 \%)$ and $4(8 \%)$. And the corresponding numbers in the TKI group were 
14(35\%), 14(35\%) and 6(15\%). Patients treated with RT were less likely to experience intracranial failure as the first failure, compared with patients treated with TKI $(P=0.091)$.

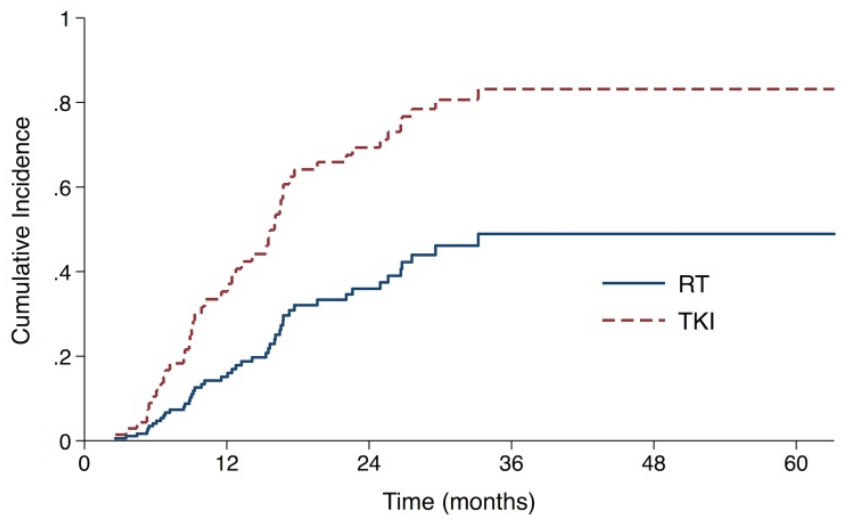

Figure 1. Cumulative incidence of intracranial progression using competing risks regression analysis in patients treated with upfront RT and epidermal growth factor receptor-TKI.

Table 2. First site of progression by treatment type.

\begin{tabular}{llll}
\hline \multirow{2}{*}{ First site of progression } & \multicolumn{3}{c}{ Treatment, N (\%) } \\
\cline { 2 - 3 } & RT $(\mathrm{n}=53)$ & TKI $(\mathrm{n}=40)$ & $P$ value \\
\hline Intracranial failure & $10(18)$ & $14(35)$ & 0.091 \\
Extracranial failure & $22(42)$ & $14(35)$ & \\
Simutaneous failure & $4(8)$ & $6(15)$ & \\
No failure & $17(32)$ & $6(15)$ & \\
\hline Abbreviations: RT, radiation therapy; TKI, tyrosine kinase inhibitor &
\end{tabular}

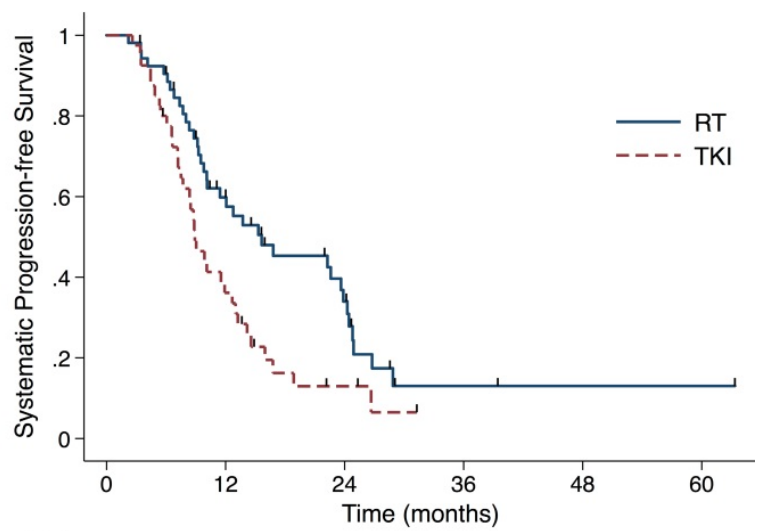

Number at risk

$\begin{array}{ccccccc}\text { RT } & 53 & 26 & 12 & 2 & 1 & 1 \\ \text { TKI } & 40 & 14 & 3 & 0 & 0 & 0\end{array}$

Figure 2. The sPFS in the RT group (median time, 15.6 months) was longer than in the TKI alone group (median time, 8.9 months) $(P=0.009)$. Abbreviation: sPFS, systemic progression-free survival; RT, radiation therapy; TKI, tyrosine kinase inhibitors.

The median OS from BM diagnosis was 35.6 months (95\% CI, 31.2-40.0 months) for the whole cohort. As shown in Figure 3A, OS did not differ significantly between the RT and the TKI group, with a median OS of 35.4 (95\% CI, 30.3-40.6 months) months and 35.8 months (95\% CI, 27.1-44.5 months), respectively $(P=0.695)$. After the Cox proportional hazards analysis, only the use of osimertinib was a protective factor to OS (HR $0.28,95 \%$ CI 0.10 to 0.78 , Table S2).

In order to analyze the reason why prolonged iPFS and sPFS failed to benefit the OS in the RT group, we looked into the salvage radiotherapy after the first progression and the length of time that patients stayed in initial TKI. For patients in the RT group, 9 out of $36(25 \%)$ received salvage RT. Among 10 patients experienced intracranial failure as first failure, $5(50 \%)$ patients received intracranial salvage RT. There were 3 and 1 patients got extracranial and simultaneous salvage RT, respectively. For patients in the TKI group, 16 out of $34(47.1 \%)$ patients got salvage RT to failure sites. 12 out of 14 (85.7\%) patients who experienced intracranial failure as the first failure received intracranial salvage RT. Also, 1 patient received simultaneous RT salvage, and 3 got extracranial salvage RT. Until the last follow-up, 31 $(58.5 \%)$ patients in the upfront RT group changed to SST and $83.9 \%(26 / 31)$ of them due to extracranial or both progressions. The corresponding number in the TKI group was $26(65.0 \%)$ and $84.6 \%(22 / 26)$. The median time from BM diagnosis to SST did not differ significantly between upfront RT group (median time, 23.6 months, 95\% CI 22.0-25.3 months) and TKI group (median time, 18.9 months, 95\% CI 8.4-29.3 months) $(P=0.862$, Figure 3B).

\section{Discussion}

Compared to the TKI group, we found that the upfront RT significantly lowered the probability of intracranial progression with SHR $0.38 \quad(P=0.006)$. However, the prolonged iPFS did not translate to the benefit of OS and time to SST. Our data showed that the salvage RT for the oligo-progression of EGFR-TKI significantly prolonged the time patients stayed in initial TKI. Even though the patients in the TKI group had poor iPFS, the salvage RT to the intracranial failure sites helped them get to the similar time to SST and OS compared to the RT group. Some retrospective studies showed the same salvage RT effectiveness. $\mathrm{Yu}$ et al. reported that patients with acquired resistance to TKI therapy achieved the PFS with 10 months and time to SST with 22 months after receiving local treatment to their oligo-progression sites[14]. Qiu et al. demonstrated that forty-six patients with oligo-progressive stage III-IV NSCLC after TKI benefited from the local therapy and continued TKI with another seven months[15]. A retrospective matched-cohort study comparing patients with oligo-progressive stage IV NSCLC showed better PFS (7.0 versus 4.1 months) and OS (28.2 versus 14.7 months) in patients receiving radiotherapy compared to patients changing to 
chemotherapy[16]. Hence, prospective clinical trials examining the efficacy of upfront RT and salvage RT on NSCLC with EGFR mutation and BM are needed.
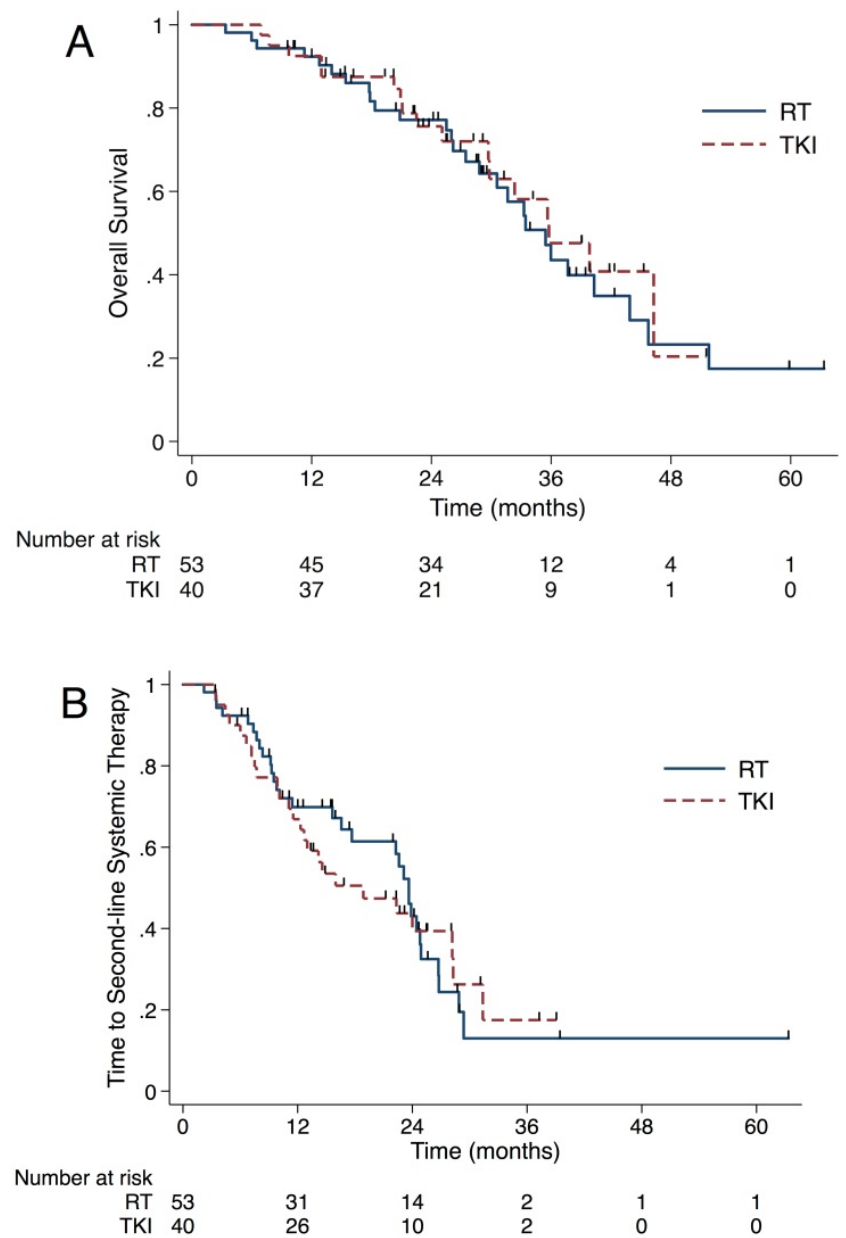

Figure 3. OS did not differ significantly between the RT and TKI group ( 35.4 vs 35.8 months, $P=0.695$ )(A). The median time from brain metastases diagnosis to SST did not differ significantly between upfront RT group and TKI group ( $23.6 \mathrm{vs}$ 18.9 months, $P=0.862$ )(B). Abbreviation: $O S$, overall survival; $R T$, radiation therapy; TKI, tyrosine kinase inhibitors; SST, second-line systematic therapy.

A few other studies also focused on the role of RT combined with TKI as upfront therapy. A retrospective study including 351 patients compared the role of SRS+TKI $(n=100)$, WBRT+TKI $(n=120)$ and TKI alone $(n=131)$ in the effectiveness of iPFS and OS[17]. It is not surprising that the patients in the SRS group had the most extended OS due to their best baseline characteristics. However, their study demonstrated that patients treated with WBRT had a trend for a lower cumulative incidence of intracranial progression $(P=0.062)$ and significantly better OS $(P=0.039)$. As to patients' characteristics, only $22 \%$ of patients in WBRT groups had extracranial metastases at the time of brain metastases. Compared to our data that $55 \%$ of patients in the RT group had extracranial metastases, the lower extracranial tumor burden in this study might help the benefit from iPFS transformed to OS. What's more, $76 \%$ of the 351 patients in this study received second-line systemic therapy right after disease progression in the last follow-up. There was no chance for salvage RT to prolong the time on initial TKI, which might be another reason that the benefit of iPFS can be transformed to OS. Jiang et al. demonstrated adding upfront WBRT to TKI compared to TKI alone didn't prolong the iPFS or OS in a retrospective study[18]. In their study, $65.9 \%$ of patients had more than 10 brain metastases and $60.0 \%$ of patients have extracranial metastases at the baseline. Compared to the previous study, the much more massive intracranial and extracranial tumor burden probably hid the benefit of iPFS from RT. A meta-analysis, which also included the above two studies, demonstrated the upfront RT helped to prolong the iPFS and OS in the same scenario[19]. It should be acknowledged that all the studies included were retrospective studies and few of them considered the role of salvage RT.

In our study, we found that the use of second-line osimertinib was the only protective factor to OS (HR $0.28,95 \% \mathrm{CI} 0.10$ to 0.78 ). It is known that osimertinib is the standard regimen in patients whose disease had progressed during first-line EGFR-TKI therapy with T790M-positive advanced NSCLC, including those with CNS metastases[20]. Osimertinib demonstrated greater penetration of the mouse blood-brain barrier than gefitinib or afatinib[21]. In the FLAURA trial, osimertinib showed benefit on PFS in patients with central nervous system metastases with median PFS 15.2 months (95\% CI 12.1-21.4 months) over standard EGFR-TKIs with median PFS 9.6 months (95\% CI 7.0-12.4 months) $(P<0.001)$ [22]. In the era that osimertinib used as the first line TKI, the role of upfront RT needs more clinical evidence to illustrate.

Although our study shed some lights on the role of upfront RT and salvage local therapy in treating EGFR mutant NSCLC with BM, several limitations should be taken into account. First, this is a retrospective study that has inherent biases despite our effort to narrow down our inclusion criteria and small cohort size. Second, we failed to perform the propensity score-matched analysis to balance the baseline of the two treatment groups due to the small number of patients. Last but not least, we did not account for the potential toxicities associated with local therapies and their impact on quality of life, such as functional independence and cognition.

\section{Conclusion}

In conclusion, upfront RT with TKI prolonged the iPFS and sPFS of the patients when compared to TKI alone. The salvage RT helped the TKI alone 
patients had a similar time on initial first-line TKI despite worse iPFS. Therefore, TKI alone will be recommended if close surveillance and timely salvage local therapy can be achieved. The best timing of intracranial radiotherapy on EGFR-mutant NSCLC with brain metastases patients remains to be verified by large-sample and risk-stratified prospective clinical trials.

\section{Supplementary Material}

Supplementary tables.

http://www.jcancer.org/v10p1985s1.pdf

\section{Acknowledgement}

This work was supported by CAMS Initiative for Innovative Medicine (grant number 2017-I2M-1-005), the National Natural Science Foundation of China (grant number 81572971) and the Non-profit Central Research Institue Fund of Chinese Academy of Medical Sciences (grant number 2018PT32011).

\section{Competing Interests}

The authors have declared that no competing interest exists.

\section{References}

1. Chen W, Zheng R, Baade PD, Zhang S, Zeng H, Bray F, et al. Cancer statistics in China, 2015. CA: a cancer journal for clinicians. 2016; 66: 115-32.

2. Siegel RL, Miller KD, Jemal A. Cancer Statistics, 2017. CA: a cancer journal for clinicians. 2017; 67: 7-30.

3. Chen Z, Fillmore CM, Hammerman PS, Kim CF, Wong KK. Non-small-cell lung cancers: a heterogeneous set of diseases. Nature reviews Cancer. 2014; 14: 535-46.

4. Barnholtz-Sloan JS, Sloan AE, Davis FG, Vigneau FD, Lai P, Sawaya RE. Incidence Proportions of Brain Metastases in Patients Diagnosed (1973 to 2001) in the Metropolitan Detroit Cancer Surveillance System. Journal of Clinical Oncology. 2004; 22: 2865-72.

5. Sperduto PW, Kased N, Roberge D, Xu Z, Shanley R, Luo X, et al. Summary report on the graded prognostic assessment: an accurate and facile diagnosis-specific tool to estimate survival for patients with brain metastases. Journal of clinical oncology : official journal of the American Society of Clinical Oncology. 2012 : 30: 419-25.

6. Li J, Bentzen SM, Renschler M, Mehta MP. Regression After Whole-Brain Radiation Therapy for Brain Metastases Correlates With Survival and Improved Neurocognitive Function. Journal of Clinical Oncology. 2007; 25: $1260-6$

7. Chang EL, Wefel JS, Hess KR, Allen PK, Lang FF, Kornguth DG, et al. Neurocognition in patients with brain metastases treated with radiosurgery or radiosurgery plus whole-brain irradiation: a randomised controlled trial. The Lancet Oncology. 2009; 10: 1037-44.

8. Martinez P, Mak RH, Oxnard GR. Targeted Therapy as an Alternative to Whole-Brain Radiotherapy in EGFR-Mutant or ALK-Positive Non-Small-Cell Lung Cancer With Brain Metastases. JAMA oncology. 2017.

9. Iuchi T, Shingyoji M, Sakaida T, Hatano K, Nagano O, Itakura M, et al. Phase II trial of gefitinib alone without radiation therapy for Japanese patients with brain metastases from EGFR-mutant lung adenocarcinoma. Lung cancer (Amsterdam, Netherlands). 2013; 82: 282-7.

10. Park SJ, Kim HT, Lee DH, Kim KP, Kim SW, Suh C, et al. Efficacy of epidermal growth factor receptor tyrosine kinase inhibitors for brain metastasis in non-small cell lung cancer patients harboring either exon 19 or 21 mutation. Lung cancer (Amsterdam, Netherlands). 2012; 77: 556-60.

11. Wu YL, Zhou C, Cheng Y, Lu S, Chen GY, Huang C, et al. Erlotinib as second-line treatment in patients with advanced non-small-cell lung cancer and asymptomatic brain metastases: a phase II study (CTONG-0803). Annals of Oncology. 2013; 24: 993-9.

12. Eisenhauer EA, Therasse P, Bogaerts J, Schwartz LH, Sargent D, Ford R, et al New response evaluation criteria in solid tumours: revised RECIST guideline (version 1.1). European journal of cancer (Oxford, England : 1990). 2009; 45: 228-47.
13. Fine JP, Gray RJ. A Proportional Hazards Model for the Subdistribution of a Competing Risk. Journal of the American Statistical Association. 1999; 94: 496-509.

14. Yu HA, Sima CS, Huang J, Solomon SB, Rimner A, Paik P, et al. Local therapy with continued EGFR tyrosine kinase inhibitor therapy as a treatment strategy in EGFR-mutant advanced lung cancers that have developed acquired resistance to EGFR tyrosine kinase inhibitors. Journal of thoracic oncology : official publication of the International Association for the Study of Lung Cancer. 2013; 8: 346-51.

15. Qiu B, Liang Y, Li Q, Liu G, Wang F, Chen Z, et al. Local Therapy for Oligoprogressive Disease in Patients With Advanced Stage Non-small-cell Lung Cancer Harboring Epidermal Growth Factor Receptor Mutation. Clin Lung Cancer. 2017; 18: e369-e73.

16. Chan OSH, Lee VHF, Mok TSK, Mo F, Chang ATY, Yeung RMW. The Role of Radiotherapy in Epidermal Growth Factor Receptor Mutation-positive Patients with Oligoprogression: A Matched-cohort Analysis. Clinical Oncology. 29: 568-75.

17. Magnuson WJ, Lester-Coll NH, Wu AJ, Yang TJ, Lockney NA, Gerber NK, et al. Management of brain metastases in tyrosine kinase inhibitor-Naive epidermal growth factor receptor-mutant non-small-cell lung cancer: a retrospective multi-institutional analysis. Journal of clinical oncology; 2017. p. 1070-7.

18. Jiang T, Su C, Li X, Zhao C, Zhou F, Ren S, et al. EGFR TKIs plus WBRT demonstrated no survival benefit other than that of TKIs alone in patients with NSCLC and EGFR mutation and brain metastases. Journal of Thoracic Oncology. 2016; 11: 1718-28.

19. Wang C, Lu X, Lyu Z, Bi N, Wang L. Comparison of up-front radiotherapy and TKI with TKI alone for NSCLC with brain metastases and EGFR mutation: A meta-analysis. Lung cancer (Amsterdam, Netherlands). 2018; 122: 94-9.

20. Mok TS, Wu YL, Ahn MJ, Garassino MC, Kim HR, Ramalingam SS, et al. Osimertinib or Platinum-Pemetrexed in EGFR T790M-Positive Lung Cancer. The New England journal of medicine. 2017; 376: 629-40.

21. Ballard P, Yates JW, Yang Z, Kim DW, Yang JC, Cantarini M, et al. Preclinical Comparison of Osimertinib with Other EGFR-TKIs in EGFR-Mutant NSCLC Brain Metastases Models, and Early Evidence of Clinical Brain Metastases Activity. Clinical cancer research : an official journal of the American Association for Cancer Research. 2016; 22: 5130-40.

22. Soria JC, Ohe $Y$, Vansteenkiste J, Reungwetwattana T, Chewaskulyong B, Lee $\mathrm{KH}$, et al. Osimertinib in Untreated EGFR-Mutated Advanced Non-Small-Cell Lung Cancer. The New England journal of medicine. 2018; 378: 113-25. 